\title{
The Vibration Map as a Tool for Diagnosis and Evaluation
}

\author{
Jorge Patricio, Fernando Schiappa and Vasco Patricio \\ Laboratorio Nacional de Engenharia Civil, Av. do Brasil, 101, 1700-066 Lisboa, Portugal
}

(Received@@@; revised@@@; accepted@@@)

\begin{abstract}
This article presents a methodology that can be used to produce vibration maps, which are caused by blasts, in order to remove rocks, in civil construction works. These maps can work in conjunction with the noise maps, providing information about the areas that are within the designated influence area by these works, and thus enabling the user to analyse and evaluate the predictable values of vibration velocity. These maps can also be used to evaluate the reasonability of the existence of complaints in the areas located in the neighbourhood of the construction sites (in the form of annoyance to persons or cosmetic damages in buildings).
\end{abstract}

\section{INTRODUCTION}

In the past few years, there has been an increase in the concerns related to vibrations, caused by several agents, such as railroad traffic, rock removal actions, by the use of explosives, or simply by the use of pylons in foundation stakes. These concerns have also been in origin of complaints, both to the entities involved in the constructions, as to the official institutions responsible by the construction works. Some of these complaints, supported by proof of patrimonial damages and psico-physiological disturbances usually proceed to judicial courts. ${ }^{\mathbf{1 , 2}}$

One instrument that is quite adequate to the evaluation, qualification and quantification of the effects of vibrations, in parallel with what has already been done in the acoustical domain, is the vibration map. Based in equipotential lines of vibration amplitude or acceleration, that can be complemented with a statistical component, which allows the input of confidence levels to the available information, it becomes possible to obtain a perspective of the effects of vibrations in buildings, and also the control of appropriate corrective measures.

The main purpose of the vibration control caused by the use of explosives in civil construction Works, is to guarantee that in the buildings near the site, the values of the parameters that quantify the predictable vibrations, do not surpass admissible limits (specially inside the buildings), which are established in the Portuguese Standard NP 2074. ${ }^{3}$

The main goal of the NP 2074 is to stipulate limitation criteria for the admissible values of vibration velocity that structures (buildings), used for dwelling, industry or services, can withstand. This criterion is related to the maximum allowable ppv (peak particle velocity), according to the following equation:

$$
v_{\max }=\frac{a \beta \gamma}{100}
$$

where $\alpha$ is the soil characteristics with the following values: 2 - in the case of coherent and rigid soils, where greater than $2000 \mathrm{~m} / \mathrm{s}, 1$ - to incoherent but compact soils, and coherent but medium rigidity soils, where 1000 and $2000 \mathrm{~m} / \mathrm{s}$, and 0.5 - to incoherent soils, such as sands, mixes of sand and stone, or very soft soils, where the specific velocity of propagation of longitudinal waves is less than $1000 \mathrm{~m} / \mathrm{s} ; \beta$ is the construction type with the following values: 0.5 - to sensitive constructions that demand special care, such as monuments, hospitals, etc., 1 - to normal constructions, and 3 - to reinforced constructions; $\gamma$ is the average number of daily solicitations with the following values: 1 - when there is less than three daily solicitations, and 0.7 - when there are more than three daily solicitations.

In Table 1 , the maximum values of velocity, in $\mathrm{mm} / \mathrm{s}$, that can be verified are displayed according to the parameters previously referred (the first number correspond to $\gamma$ equal to 0.7 and the second ones to $\gamma$ equal to 1 ).

Therefore, we can focus this type of control in two important aspects: 1) guarantee that the limits established are not surpassed in all of the area close to the construction site in study; and 2) enable the reconstruction of the vibration values originated by any blasting.

The values of vibration velocities that can occur in buildings or any other location under analysis close to the construction site, must be estimated, and based on the uncertainty associated with these estimated values (statistical analysis), the maximum limits must also be calculated. This way, it is possible, by means of a simple extrapolation, to know the effect of each blasting in each point that has been considered for analysis.

The use of this type of statistical analysis will also enable the construction of a vibration map, with which is possible to emphasise the influence areas, regarding vibrations caused by the explosive blasts, and this way obtaining a knowledge about the vibration field that has been established during the course of the works. In the same way, these maps can also be used to judge the existence of complaints (people's annoyance, and cosmetic damages in buildings).

The prediction of the vibration velocities can be made using the Johnson (or Medvedev) formula, which has to be adjusted to each study location..$^{4-7}$ This adjustment must always be made, because in reality the soils aren't homogeneous, neither equal from zone to zone. Therefore, the adjustment of this formula must be made based in measurements taken in different directions of propagation, in order to characterise the respective soil. Because the adjustment is usually made by recurring to a reduced number of measurements

(pp 31-35) 